\title{
A GENERALIZED FRATTINI SUBGROUP OF A FINITE GROUP
}

\section{PRABIR BHATTACHARYA}

Department of Computer Science University of Nebraska - Lincoln Lincoln, NE 68588-0115, U.S.A.

\section{N.P. MUKHERJEE}

School of Computer and System Sciences Jawaharlal Nehru University New Delhi 110067, India

(Received December 1, 1987)

ABSTRACT. For a finite group $G$ and an arbitrary prime $p$, let $S_{p}(G)$ denote the intersection of all maximal subgroups $M$ of $G$ such that [G:M] is both composite and not divisible by $p$; if no such $M$ exists we set $S_{p}(G)=G$. Some properties of $G$ are considered involving $S_{p}(G)$. In particular, we obtain a characterization of $G$ when each $M$ in the definition of $S_{P}(G)$ is nilpotent.

KEY WORDS AND PHRASES. Solvable group, Nilpotent group, Supersolvable group, Frattini subgroup.

1980 AMS SUBJECT CLASSIFICATION CODES. 20D 10, 20D5

1. INTRODUCTION.

It is an interesting problem to investigate the relationships between the structure of a finite group $G$ and the properties of the maximal subgroups of $G$. This has been studied by several people (e.g. [4], [5]). In [2] and [7-8] we have considered the family of maximal subgroups whose indices are composite and co-prime to a given prime. In this note we obtain further results in this direction. All groups considered are finite. A maximal subgroup $M$ of a group $G$ will be sometimes denoted by $M<\cdot G$. A maximal subgroup $M$ of $G$ of composite index will be called c-maximal.

2. THE SUBGROUP $s_{p}(G)$.

Let $G$ be a group and $P$ any prime. Consider the family of subgroups of $G$ :

$$
\mathrm{J}=\left\{\mathrm{M}: \mathrm{M} \text { is c-maximal, }[\mathrm{G}: \mathrm{M}]_{\mathrm{p}}=1\right\}
$$

Define $S_{p}(G)=n\{M: M \varepsilon J\}$, if $J$ is empty then set $s_{p}(G)=G$. This subgroup was introduced by us and several results have been obtained in [2] and [7]. We remark that $S_{P}(G)$ is a characteristic subgroup containing the Frattini subgroup $\Phi(G)$.

Our first result is motivated by Rose [9] where it was proved that if every non-normal, maximal subgroup of a group is nilpotent then the group is solvable. This result was extended by us ([2, Theorem 1.1]). We now obtain a further result in this direction. 
THEOREM 2.1. Let $\mathrm{p}$ be the largest prime divisor of the order of a group $G$. Suppose that each subgroup in the family $J$ (see above for the definition) is nilpotent. Then

(i) either, $G$ is $p$-nilpotent or, there exists a normal p-subgroup $P_{0}$ of $G$ such that $\mathrm{G} / \mathrm{P}_{0}$ is $\mathrm{P}$-nilpotent.

(ii) if $1_{p}(G)$ denotes the p-length of $G$ then $1_{p}(G) \leq 2$.

(Note: It follows directly from [2, Theorem 1.1] that $G$ is solvable in this case).

PROOF: (i) we distinguish two cases:

Case 1: G has no normal p-subgroup. Let $P$ be a Sylow p-subgroup of $G$. Then $N_{G}(P) \neq G$ and choose $M<G$ such that $N_{G}(P) \leq M$. If [G: $\left.M\right]$ is a prime $q$, say, then it is easy to see that $q>p$, an impossibility, thus [G: $M$ ] is composite and clearly $[G: M]_{P}=1$. So $M \in J$ implying that $M$ is nilpotent. Therefore $M=$ $N_{G}(P)$. Let $P_{0}$ be a nontrivial characteristic subgroup of $P$. As $G$ has no normal p-subgroup, $N_{G}\left(P_{0}\right)=N_{G}(P)=M$. Consequently $M$ induces only $p$-automorphism on $P_{0}$ and so by Thompson [10] $G$ is p-nilpotent.

Case 2: G has a normal p-subgroup. Let $P_{0}$ be a normal p-subgroup of $G$ of the largest possible order. If $P_{0}$ is a sylow $p$-subgroup of $G$ then trivially $G / P_{0}$ is p-nilpotent. So, assume that $P_{0}$ is not a sylow p-subgroup. We use induction on $|G|$. We note that $p$ is the largest prime dividing $\left|G / P_{0}\right|$. It is easy to see that $G / P_{0}$ satisfies the hypothesis of the theorem and $G / P_{0}$ has no normal p-subgroup. So by induction hypothesis $G / P_{0}$ is $p$-nilpotent. Thus the proof of (i) is complete and (ii) follows now readily.

Our next result illustrates how under certain conditions the supersolvability of a group is controlled by the structure of certain groups of smaller orders.

THEOREM 2.2. For a group $G$ and any prime $p$, if $\left|S_{p}(G)\right|$ is co-prime to $p$, then $G$ is supersolvable $\Leftrightarrow G / S_{P}(G)$ is supersolvable.

PROOF: The case $\Rightarrow$ is trivial and we consider now the $<=$ case.

If every maximal subgroup of $G$ is of prime index then $G$ is supersolvable by a well known result of Huppert and so $S_{p}(G)$ is supersolvable. Now let $M$ be $a$ c-maximal subgroup of $G$. If $M$ does not contain $S_{p}(G)$, then $G=M S_{p}(G)$ and so $[G: M]_{P}=1$ since by hypothesis $\left|S_{P}(G)\right|$ is a $P^{\prime}$-number. Consequently $M \varepsilon J$ and so $S_{P}(G) \leq M$, a contradiction. Thus $S_{P}(G)$ is contained in every c-maximal subgroup of $G$ and so $S_{P}(G)$ is contained in $L(G)$, the intersection of all c-maximal subgroups of G. Now by [1] (see [2] for a published proof) L(G) is supersolvable and so the result now follows.

A group $G$ is called a sylow tower group of supersolvable type if (i) $p_{1}>p_{2}$ $\ldots>P_{k}$ are all the prime divisors of $|G|$ and $P_{i}$ is a sylow $P_{i}$-subgroup of $G$ and (ii) $\mathrm{P}_{1} \mathrm{P}_{2} \cdots \mathrm{P}_{\mathrm{k}}<\mathrm{G}, \mathrm{l} \leq \mathrm{i} \leq \mathrm{k}$.

THEOREM 2.3. Let $q$ be the largest prime divisor of a group $G$ and assume that $S_{q}(G)=G$. (In other words, the family $J$ in the definition of $S_{q}(G)$ is empty). Then $G$ is a Sylow tower group of supersolvable type.

PROOF. We use induction on $|G|$. If $Q$ is a sylow $q$-subgroup of $S_{q}(G)$ then by [7, Proposition 5] $Q<G$. Consider the following two families of subgroups: 


$$
\begin{gathered}
\mathrm{J}=\left\{M: M \text { is c-maximal in } G,[G: M]_{\mathrm{q}}=1\right\} \\
\mathrm{J}_{1}=\left\{M / Q: M / Q \text { is c-maximal in } G / Q,[G / Q: M / Q]_{\mathrm{q}}=1\right\}
\end{gathered}
$$

Since $S_{q}(G)=G, J$ is empty. This implies that $J_{1}$ is also empty. For, if $J_{1}$ is nonempty and $M / Q$ belongs to $J_{1}$ then clearly $M \in J$, contradicting the fact that $J$ is empty. Hence $S_{q}(G / Q)=G / Q$. This implies that if $M / Q$ is an arbitrary maximal subgroup of $G / Q$ then clearly $[G / Q: M / Q]_{q}=1$ and $[G / Q: M / Q]$ must be a prime. Thus every maximal subgroup of $G / Q$ is of prime index. So, by a well known result of Huppert $G / Q$ is supersolvable. Hence $G$ is a sylow tower group of supersolvable type.

If $G$ is supersolvable then every maximal subgroup of $G$ is of prime index by a well known result of Huppert, and so $S_{p}(G)=G$. Thus, if $G$ is supersolvable then for $H \leq G$, we have that $S_{P}(G) \cap H=S_{P}(H)$. A simple example will show that the converse is not always true. (Take $G=A_{4}$ and $p=2$. Here $S_{2}(H)=H$ for every subgroup $\mathrm{H}$ but $\mathrm{A}_{4}$ is not supersolvable). However, we have the following partial converse:

PROPOSITION 2.4. Let $\mathrm{p}$ be the largest prime dividing the order of a group $\mathrm{G}$. Suppose that $S_{P}(G) \cap H=S_{P}(H)$ for every subgroup $H$ of $G$. Then $G$ is a Sylow tower group of supersolvable type.

PROOF: Let $Q$ be any Sylow $q$-subgroup of $G$ where $q$ is any prime dividing $|G|$. By hypothesis, $S_{P}(G) \cap Q=S_{P}(Q)$. Further since any maximal subgroup of $Q$ is of prime index in $Q, S_{p}(Q)=Q$ irrespective of the fact that $p$ may or may not be equal to $q$. Thus $S_{p}(G)$ contains every Sylow q-subgroup of $G$ for every prime $q$ dividing $|G|$. Therefore $S_{P}(G)=G$. The result now follows by applying Theorem 2.3.

We omit the proof of the following standard result:

LEMMA 2.5. Let $G$ be a supersolvable group in which for every maximal subgroup $M,[G: M]=p$ where $p$ is a fixed prime. Then $G$ is a $p$-group.

We now prove:

PROPOSITION 2.6. Let $\mathrm{p}$ be the largest prime dividing the order of a group $G$. (i) Assume that $[G: M]_{p}=1$ implies that $[G: M]$ is a prime for any $M<\cdot G$. Then $G$ is a Sylow-tower group of supersolvable type. Further if $P$ is a sylow p-subgroup of $G$ then $P<G$ and $G / P$ is supersolvable.

(ii) Let $q$ be any prime such that $q$ is not equal to $p$. Assume that $[G: M]_{p}=1$ implies that $[G: M]=q$ for any $M<\cdot G$ and furthermore $\left[G: M_{1}\right]_{q}=1$ implies that $\left[G: M_{1}\right]=p$ for any $M_{1}<\cdot G$. Then $G$ is supersolvable.

PROOF: We omit the proof of (i) which is a direct consequence of Theorem 2.3. Now consider ( $i i)$. If $P$ is a sylow p-subgroup of $G$ then by ( $i), P<G$ and $G / P$ is supersolvable. Now $q$ divides $|G / P|$. Suppose if possible $M / P<\cdot G / P$ such that $[\mathrm{G} / \mathrm{P}: \mathrm{M} / \mathrm{P}]_{\mathrm{q}}=\mathrm{i}$. Then $[\mathrm{G}: \mathrm{M}]_{\mathrm{q}}=1$ and so by hypothesis $[\mathrm{G}: \mathrm{M}]=\mathrm{p}$ which is impossible since $M$ contains $P$. Thus no maximal subgroup of $G / P$ has index co-prime to $q$ and since $G / P$ is supersolvable, this gives, by using a well known result of Huppert, that every maximal subgroup of $G / P$ is of prime index, and so has index $q$. By Lemma 2.5 it now follows that $G / P$ is a $q-g r o u p$ and so $|G|$ is of 
the form $p^{\alpha} q^{\beta}$. By using a well-known result of Burnside, $G$ is solvable. We now show that $G$ is supersolvable. Suppose if possible that there exists a maximal subgroup $M$ such that [G:M] is divisible by both $p$ and $q$. Then $G=P$ and it follows that $[G: M]=|P| /|P \cap M|$ is a power of $p$, a contradiction. Therefore, for any $M<\cdot G$, we have that the index of $M$ in $G$ is either co-prime to $p$, or co-prime to $q$. Consequently, by the hypothesis it follows that every maximal subgroup of $G$ is of prime index and hence $G$ is supersolvable by using a well-known result of Huppert.

REMARK: Under the hypothesis of Theorem 2.6 (ii) it might be tempting to conjecture that $G$ is nilpotent. However, $S_{3}$ satisfies the hypothesis but is not nilpotent.

\section{REFERENCES}

1. H.C. Bhatia, A generalized Frattini subgroup of a finite group. Ph.D. thesis, Michigan State University, East Lansing, 1972.

2. P. Bhattacharya and N.P. Mukherjee, A family of maximal subgroups containing the Sylow subgroups and some solvability conditions, Arch. Math. 45, 390-397 (1985).

3. P. Bhattacharya and N.P. Mukherjee, On the intersection of a class of maximal subgroups of a finite group II, J. Pure and Applied Algebra 42 , $117-124$ (1986).

4. W.E. Deskins, On Maximal Subgroups. In : First Sympos. Pure Math., American Math. Soc. Providence, (1959), 100-104.

5. W. Gaschutz, Uber die $\Phi$ - Untergruppe endlicher Gruppen. Math. Zeit. $\underline{58}$, 160-170 (1953).

6. B. Huppert, Endliche Gruppen I Berlin 1967.

7. N.P. Mukherjee and P. Bhattacharya, On the intersection of a class of maximal subgroups of a finite group, Canadian J. Math. 39, 603-611 (1987).

8. N.P. Mukherjee and P. Bhattacharya, On the normal index of a finite group, Pacific J. of Math. 132, 143-149 (1988).

9. J. Rose, The influence on a group of its abnormal structure, J. London Math. Soc. 40, 348-361 (1965).

10. J. Rose, On finite insoluble groups with nilpotent maximal subgroups. J. Algebra 48, 182-196 (1977).

11. J.G. Thompson, Normal p-complements for finite groups, J. Algebra 1, 43-46 (1964). 


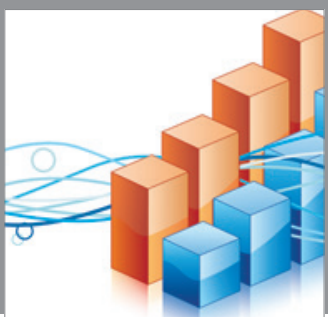

Advances in

Operations Research

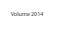

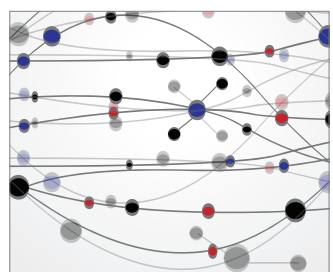

\section{The Scientific} World Journal
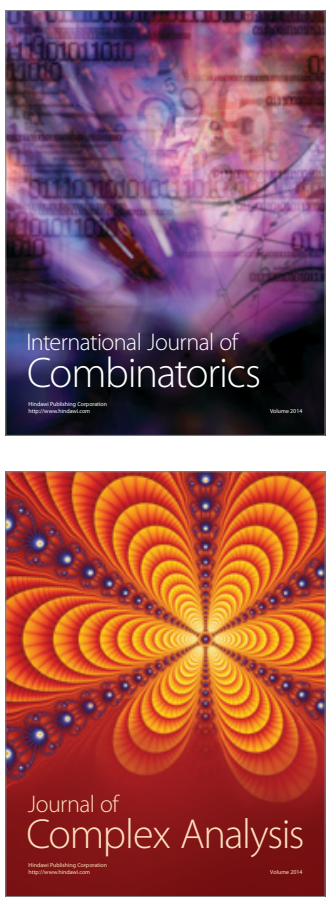

International Journal of

Mathematics and

Mathematical

Sciences
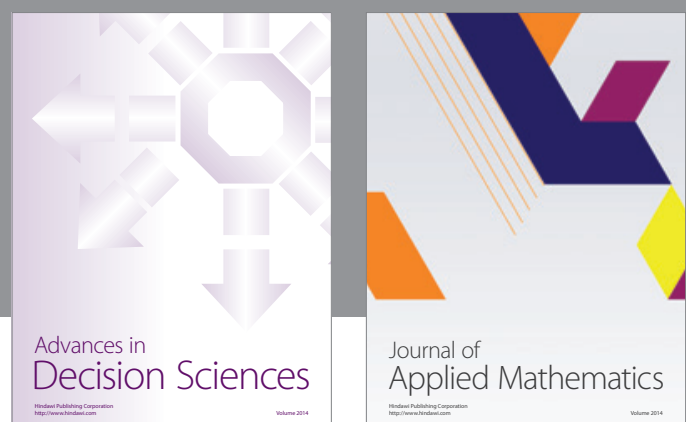

Journal of

Applied Mathematics
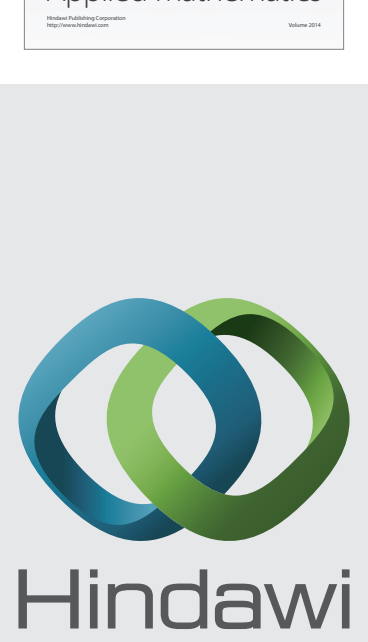

Submit your manuscripts at http://www.hindawi.com
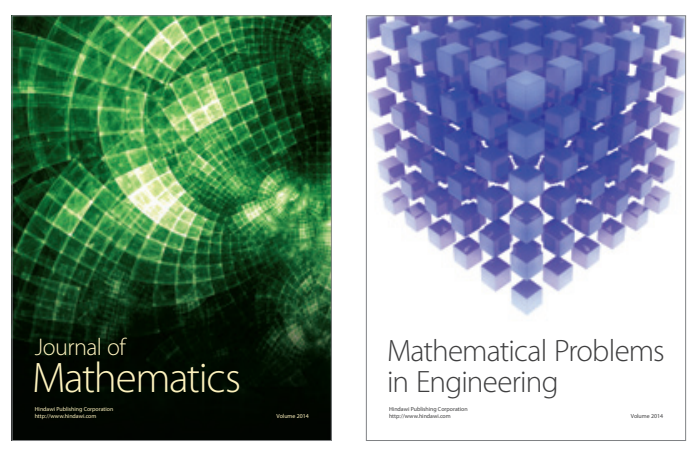

Mathematical Problems in Engineering
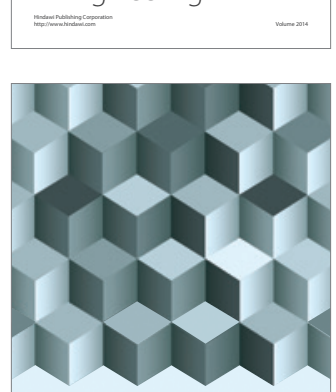

Journal of

Function Spaces
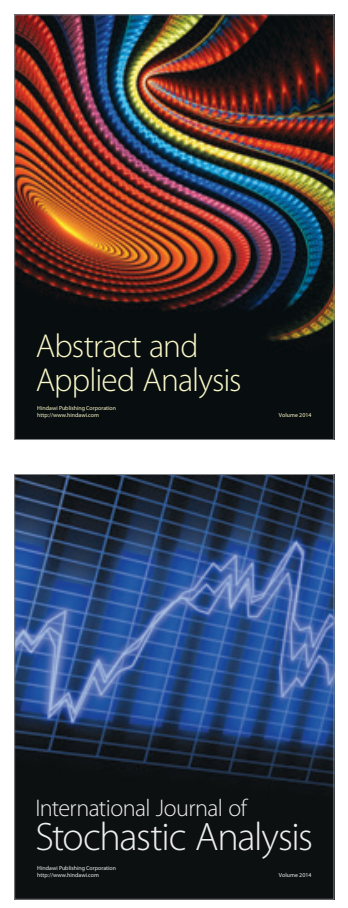

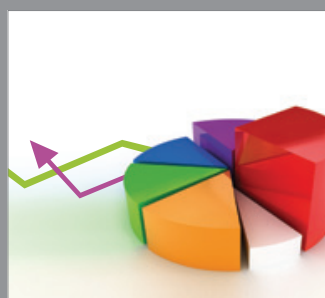

ournal of

Probability and Statistics

Promensencen
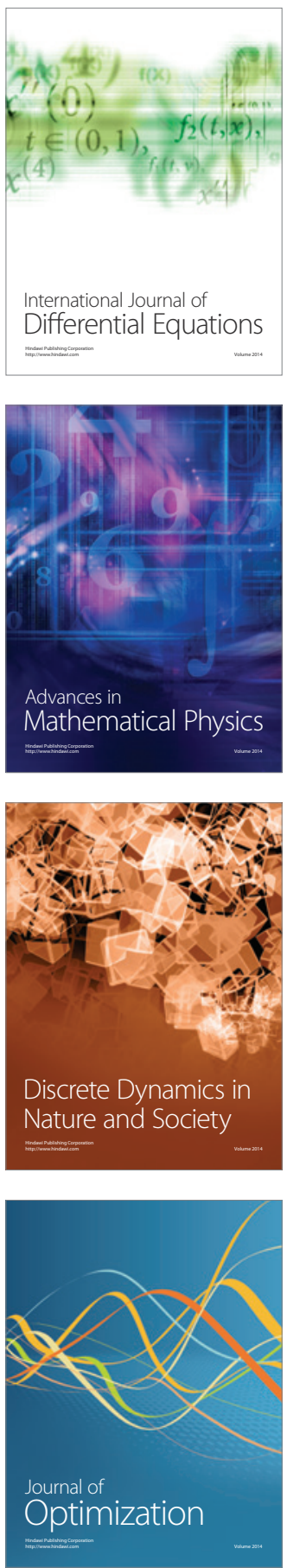